Bull. Soc. math. France

130 (4), 2002, p. 493-506

\title{
INVARIANCE OF GLOBAL SOLUTIONS OF THE HAMILTON-JACOBI EQUATION
}

\author{
BY EZEQUiEL MADERNA
}

\begin{abstract}
We show that every global viscosity solution of the Hamilton-Jacobi equation associated with a convex and superlinear Hamiltonian on the cotangent bundle of a closed manifold is necessarily invariant under the identity component of the group of symmetries of the Hamiltonian (we prove that this group is a compact Lie group). In particular, every Lagrangian section invariant under the Hamiltonian flow is also invariant under this group.

RÉSUMÉ (Invariance des solutions globales de l'équation de Hamilton-Jacobi)

On prouve que toute solution globale de viscosité de l'équation de Hamilton-Jacobi associée à un hamiltonien convexe et superlinéaire sur le fibré cotangent d'une variété fermée est toujours invariante sous l'action de la composante neutre du groupe de symétries du hamiltonien (on montre que ce groupe est un groupe de Lie compact). En particulier, toute section lagrangienne du fibré cotangent qui est preservée par le flot hamiltonien doit être invariante sous cette action.
\end{abstract}

Texte reçu le 4 avril 2001, accepté le 23 mai 2001

Ezequiel Maderna, Unité de Mathématiques Pures et Appliquées, École Normale Supérieure de Lyon, 46 allée d'Italie, 69364 Lyon Cedex 07 (France) et Instituto de Matemática y Estadística, Prof. Rafael Laguardia, Facultad de Ingeniería, Universidad de la República, Montevideo (Uruguay) • E-mail : eze@fing.edu.uy Url : http://www.fing.edu.uy\imerl

2000 Mathematics Subject Classification. - 49L25, 37J50, 53D12, 70H20.

Key words and phrases. - Hamilton-Jacobi, Lagrangian, symmetries. 


\section{Introduction}

Let $M$ be a closed manifold, and let $H: T^{*} M \rightarrow \mathbb{R}$ be a $\mathcal{C}^{\infty}$ Hamiltonian that is $\mathcal{C}^{2}$-strictly convex and superlinear on the fibers of the cotangent bundle $\pi^{*}: T^{*} M \rightarrow M$.

In [3], generalizing work by Lions, Papanicolau \& Varadhan, Fathi proved the existence of global viscosity solutions, also called weak KAM solutions, of the Hamilton-Jacobi equation

$$
H\left(x, \mathrm{~d}_{x} u\right)=c
$$

and that these solutions only exist for the value $c=c(L)$, which equals Mañé's critical value of the associated Lagrangian. The latter can also be characterized in terms of Mather's minimizing measures (see [7], [8]). The solutions are given modulo constants by the fixed points of the Lax-Oleinik semigroups $T_{t}^{-}$and $T_{t}^{+}$ (see below for the definition of $T_{t}^{-}$and $T_{t}^{+}$). Now let $\mathcal{S}_{-}$and $\mathcal{S}_{+}$be the set of weak KAM solutions of $T_{t}^{-}$and $T_{t}^{+}$respectively. One has that $\mathcal{S}_{-} \cap \mathcal{S}_{+}=\mathcal{S}$, the set of classical solutions of the Hamilton-Jacobi equation, i.e. of class $\mathcal{C}^{1}$.

The weak KAM solutions are very useful in the study of the dynamics of the Hamiltonian vector field $X_{H}$ associated with $H$ (see also [1], [2]).

We will denote by $\Gamma_{H}$ the group of diffeomorphisms of $M$ of class $\mathcal{C}^{1}$ that preserve $H$, more precisely

$$
\Gamma_{H}=\left\{g \in \operatorname{Diff}^{1}(M) ; H(g(x), p)=H\left(x, p \circ \mathrm{d}_{x} g\right), \forall x \in M, p \in T_{g(x)}^{*} M\right\}
$$

endowed with the topology of uniform convergence. Let $\Gamma_{H}^{0}$ be the identity component of $\Gamma_{H}$. We shall prove in Section 4 that $\Gamma_{H}$ is a compact Lie group.

In [5], the proof of the existence of weak KAM solutions is generalized to the case when $M$ is not necesarily compact, with the additional hypothesis of uniform superlinearity (with respect to a complete Riemannian metric) of the Hamiltonian and its associated Lagrangian. In [5], we also show the existence of $\Gamma_{H}$-invariant weak KAM solutions for values of the constant greater or equal than a certain value $c_{\text {inv }} \geq c(L)$. It follows that if $M$ is compact $c_{\text {inv }}=c(L)$. We will prove later these facts in a slightly simplified way using compactness.

On the other hand, if $M$ is not compact the inequality $c_{\text {inv }} \geq c(L)$ could be strict. This follows from the examples given by G. \& M. Paternain [10] on the universal cover of closed surface of genus 2 .

In this paper we show:

Theorem 1. - Let $M$ be a closed manifold, and let $H: T^{*} M \rightarrow \mathbb{R}$ be a $\mathcal{C}^{\infty}$ Hamiltonian that is convex and superlinear on the fibers of the cotangent bundle of $M$. If $\Gamma_{H}$ is the symmetry group of $H$, then every weak KAM solution of $H$ is $\Gamma_{H}^{0}$-invariant, where $\Gamma_{H}^{0}$ denote the identity component of $\Gamma_{H}$.

In general, Hamiltonians have trivial symmetry groups like general Riemannian metrics which usually have trivial isometry groups. However we find

TOME $130-2002-\mathrm{N}^{\mathrm{O}} 4$ 
Hamiltonian systems with symmetries quite often in the applications, and these symmetries are very useful for a detailed study of the system. If the dimension of $\Gamma_{H}$ is sufficiently large we can find all weak KAM solutions by integration, as we will see in the case when $H$ is the Hamiltonian of the mechanical system determined by the motion of a particle on the $n$-sphere $\mathbb{S}^{n} \subset \mathbb{R}^{n+1}$ under the effect of a potential $U(x)=x_{n+1}$. In this case we can reduce the problem to finding the weak KAM solutions of the pendulum on the circle.

In particular, our result applies to the solutions of the Hamilton-Jacobi equation of class $\mathcal{C}^{2}$ that correspond to exact Lagrangian sections of $T^{*} M$ invariant under the Hamiltonian flow associated with $H$. To reduce the study of Lagrangian sections to exact ones, we shall recall in Section 5 that given any cohomology class in $H^{1}(M, \mathbb{R})$ there exist a closed $\Gamma_{H}$-invariant 1 -form that represent the class. Combining this result with theorem 1 we obtain the following corollary whose proof will also be given in Section 5:

Corollary 2. - Every Lagrangian section of $T^{*} M$ invariant under the Hamiltonian flow of $H$ is also invariant under $\Gamma_{H}^{0}$.

\section{Weak KAM solutions and Mather's set}

Before giving the proof of Theorem 1, we briefly recall the properties of the weak KAM solutions which we will use. The details of the proofs can be found in [3] and [4].

Let us introduce initially the Lagrangian corresponding to $H$ like its convex dual on the tangent bundle of $M$ :

$$
L: T M \longrightarrow \mathbb{R}, \quad L(x, v)=\sup \left\{p(v)-H(x, p) ; p \in T_{x}^{*} M\right\} .
$$

It is well-known that $L$ is also of $\mathcal{C}^{\infty}$ class, strictly convex and superlinear on the fibers, i.e. its second derivative $\partial^{2} L / \partial v^{2}$ is definite positive everywhere and for all $K \in \mathbb{R}$ there exists a constant $C_{K}$ such that

$$
\forall(x, v) \in T M, \quad L(x, v) \geq K\|v\|+C_{K} .
$$

The Legendre transform $\mathcal{L}: T M \rightarrow T^{*} M$,

$$
\mathcal{L}(x, v)=\left(x, \frac{\partial L}{\partial v}(x, v)\right),
$$

is a diffeomorphism which conjugates the Euler-Lagrange flow defined by $L$ on $M$, which is denoted $\phi_{t}^{L}$, with the Hamiltonian flow of $H$.

The action of $L$ on a piecewise $\mathcal{C}^{1}$ curve $\gamma:[a, b] \rightarrow M$ is as usual

$$
A_{L}(\gamma)=\int_{a}^{b} L(\gamma(s), \dot{\gamma}(s)) \mathrm{d} s
$$

BULletin DE LA SOCIÉtÉ MATHÉMATIQUE DE FRANCE 
We will say that a function $u: M \rightarrow \mathbb{R}$ is dominated by $L+c$ (for certain value of $c \in \mathbb{R}$ ) and we will write

$$
u \prec L+c
$$

if for each piecewise $\mathcal{C}^{1}$ curve $\gamma:[a, b] \rightarrow M$ we have

$$
u(\gamma(b))-u(\gamma(a)) \leq A_{L}(\gamma)+c(b-a) .
$$

From the superlinearity of $L$ it is easy to deduce that dominated functions are Lipschitz, with a Lipschitz constant which only depends, once fixed the metric on $M$, on the constant $c$ and the Lagrangian; in particular, in accordance with the Rademacher's theorem (see [11]), they are differentiable almost everywhere.

The main reason we are interested in dominated functions is that they constitute a suitable space where Lax-Oleinik's semigroups of operators can be studied. In this way, we will obtain weak KAM solutions. Since we already know that the solutions are dominated by $L+c(L)$, where $c(L)$ is the critical value of $L$, we can directly introduce the space

$$
\mathbb{H}=\left\{u \in \mathcal{C}^{0}(M, \mathbb{R}) ; u \prec L+c(L)\right\} .
$$

On this space we can define the non linear operators $u \mapsto T_{t}^{-} u, u \mapsto T_{t}^{+} u$ for each $t \geq 0$

$$
T_{t}^{-} u(x)=\inf _{\gamma \in \mathcal{C}^{-}}\left\{u(\gamma(0))+A_{L}(\gamma)\right\}, \quad T_{t}^{+} u(x)=\sup _{\gamma \in \mathcal{C}^{+}}\left\{u(\gamma(t))-A_{L}(\gamma)\right\},
$$

where

$$
\begin{aligned}
& \mathcal{C}^{-}=\left\{\gamma:[0, t] \rightarrow M ; \text { piecewise } \mathcal{C}^{1} \text { with } \gamma(t)=x\right\}, \\
& \mathcal{C}^{+}=\left\{\gamma:[0, t] \rightarrow M \text {; piecewise } \mathcal{C}^{1} \text { with } \gamma(0)=x\right\} .
\end{aligned}
$$

From the definition it follows the semigroup property

$$
T_{t}^{-} \circ T_{s}^{-}=T_{t+s}^{-} \text {for all } t, s \geq 0,
$$

and that

$$
T_{t}^{-}(u+c)=T_{t}^{-}(u)+c \text { for all } c \in \mathbb{R} .
$$

On the other hand, it is clear that $u+c \in \mathbb{H}$ whenever $u \in \mathbb{H}$; this allows us to define the quotient semigroup $\widehat{T}_{t}^{-}$acting on $\widehat{\mathbb{H}}$, the quotient set of $\mathbb{H}$ by the space of constant functions, by

$$
\widehat{T}_{t}^{-}[u]=\left[T_{t}^{-} u\right] .
$$

Analogously, we can define the quotient semigroup $\widehat{T}_{t}^{+}$.

Definition 3 (Weak KAM solution). - We say that $u \in \mathbb{H}$ is a global viscosity solution of the Hamilton-Jacobi equation, or a weak KAM solution, if

$$
\widehat{T}_{t}^{-}[u]=[u] \quad \text { or } \quad \widehat{T}_{t}^{+}[u]=[u] \text { for all } t \in \mathbb{R} .
$$

We call $\mathcal{S}_{-}$and $\mathcal{S}_{+}$respectively the sets defined by the above relations.

TOME $130-2002-\mathrm{N}^{\mathrm{O}} 4$ 
The existence of these solutions is obtained in [3] through the application of the fixed point theorem of Schauder and Tykhonov; this requires to show the continuity of the semigroups and the compactness of the convex $\widehat{\mathbb{H}}$. In the same article, it is shown that the relations which define $\mathcal{S}_{-}$and $\mathcal{S}_{+}$sets, are equivalent to

$$
T_{t}^{-} u=u-c(L) t Q \quad \text { and } \quad T_{t}^{+} u=u+c(L) t, \quad \forall t \geq 0,
$$

and that weak KAM solutions verify the Hamilton-Jacobi equation at every point where they are differentiable.

Weak KAM solutions are also characterized by the fact of being dominated by $L+c(L)$ and by the existence of certain curves on which their variation is maximal; more precisely,

Proposition 4 (Fathi [3]). - A function $u: M \rightarrow \mathbb{R}$ is in $\mathcal{S}_{-}$if and only if:

a) $u \prec L+c(L)$, where $c(L)$ is the critical value of $L$,

b) for all $x \in M$ there exists an extremal of $L, \gamma_{x}:(-\infty, 0] \rightarrow M$ with $\gamma_{x}(0)=x$, and such that for all $t \geq 0$ we have

$$
u(x)-u\left(\gamma_{x}(-t)\right)=\int_{-t}^{0} L\left(\gamma_{x}(s), \dot{\gamma}_{x}(s)\right) \mathrm{d} s+c(L) t
$$

Moreover, the set of differentiability points of a function satisfying a) contains the points $x \in M$ for which there exists $\epsilon>0$ and an extremal $\gamma:[-\epsilon, \epsilon] \rightarrow M$, such that $\gamma(0)=x$ and

$$
u(\gamma(\epsilon))-u(\gamma(-\epsilon))=\int_{-\epsilon}^{\epsilon} L(\gamma(s), \dot{\gamma}(s)) \mathrm{d} s+2 \epsilon c(L)
$$

The characterization of the functions in $\mathcal{S}_{+}$is analogous, it is enough to replace the curves of the condition b) by curves of the form $\gamma_{x}:[0,+\infty) \rightarrow M$ with $\gamma_{x}(0)=x$ along which the equality is satisfied.

Let now $\mu$ be a Borel measure on $T M$, invariant under the Euler-Lagrange flow, and let $u: M \rightarrow \mathbb{R}$ be a $(L+c)$-dominated function. Because of invariance of $\mu$ we have

$$
\int_{T M}\left(u \circ \pi \circ \phi_{1}^{L}-u \circ \pi\right) \mathrm{d} \mu=0
$$

where $\pi: T M \rightarrow M$ is the canonical projection of the tangent bundle. If one applies for each $v \in T M$, the domination of $u$ by $L+c$ to the curve $t \mapsto \pi \circ \phi_{s}^{L}(v)$ with $t$ varying in $[0,1]$, it results from it that

$$
c+\int_{T M} \int_{0}^{1} L \circ \phi_{s}^{L} \mathrm{~d} s \mathrm{~d} \mu \geq 0 .
$$

BULletin DE LA SOCIÉtÉ MATHÉMATIQUE DE FRANCE 
By reversing the order of integration and once again owing to the fact that $\mu$ is invariant, it results that

$$
\int_{T M} L \mathrm{~d} \mu \geq-c
$$

In addition, if $u \prec L+c(L)$ is a solution in $\mathcal{S}_{-}$, and $\gamma_{x}$ one of the extremals which is associated to him by Proposition 4 , one can build a $\phi_{t}^{L}$-invariant probability measure on $T M$, supported on the $\alpha$-limit set of $\gamma_{x}$; it is enough to take a weak limit when $t \rightarrow-\infty$ of the probability measures $\mu_{t}$ defined by

$$
\int f \mathrm{~d} \mu_{t}=-\frac{1}{t} \int_{t}^{0} f\left(\gamma_{x}(s)\right) \mathrm{d} s
$$

for $f: T M \rightarrow \mathbb{R}$ a continuous function. It is easy to note that the measures $\mu$ thus built satisfy

$$
\int_{T M} L \mathrm{~d} \mu=-c(L)
$$

This indeed shows well the next characterization of the critical value of the Lagrangian:

1) $c(L)$ is the least value of $c \in \mathbb{R}$ such that the set $\{f: M \rightarrow \mathbb{R} ; f \prec L+c\}$ is not empty.

2) $c(L)=-\inf \int_{T M} L \mathrm{~d} \mu$, where the infimum is taken over all probability measures invariants by the Euler-Lagrange flow.

According to Mather [8], a measure $\mu$ is said to be minimizing if it is Borel probability measure $\mu$, invariant by the Euler-Lagrange flow, and

$$
\int_{T M} L \mathrm{~d} \mu=-c(L)
$$

The Mather set $\widetilde{\mathcal{M}}$ is the closure of the union of the supports of all minimizing measures; it is thus compact, invariant by $\phi_{t}^{L}$, and it contains the $\alpha$-limit sets of curves $\gamma_{x}$ referred to above. If $(x, v) \in \widetilde{\mathcal{M}}$, and $u$ be a solution in $\mathcal{S}_{-}$or $\mathcal{S}_{+}$, then for all $t, t^{\prime} \in \mathbb{R}$ we have

$$
u\left(\pi \phi_{t}^{L}(x, v)\right)-u\left(\pi \phi_{t^{\prime}}^{L}(x, v)\right)=\int_{t^{\prime}}^{t} L\left(\phi_{s}^{L}(x, v)\right) \mathrm{d} s+c(L)\left(t-t^{\prime}\right) .
$$

This proves that $u$ is differentiable on $\mathcal{M}=\pi(\widetilde{\mathcal{M}})$ and that if $(x, v) \in \widetilde{\mathcal{M}}$ then $\mathrm{d}_{x} u=\mathcal{L}(x, v)$ (see [3, Prop. 3]); in particular $\pi: \widetilde{\mathcal{M}} \rightarrow \mathcal{M}$ is one to one.

\section{Proof of Theorem 1}

At first we show that $\mathcal{M}=\pi(\widetilde{\mathcal{M}})$ is invariant under $\Gamma_{H}$, and that the restrictions of solutions in $\mathcal{S}_{-}$to $\mathcal{M}$ are $\Gamma_{H}^{0}$-invariant (the proof for the case of solutions in $\mathcal{S}_{+}$is analogous). Actually, it is evident that the transformations

TOME $130-2002-\mathrm{N}^{\mathrm{O}} 4$ 
which preserve $H$ also preserve $L$; to be more precise, for all $g \in \Gamma_{H}$, and for all $(x, v) \in T M$ we have

$$
L\left(g(x), \mathrm{d}_{x} g(v)\right)=L(x, v) .
$$

Then, $A_{L}(\gamma)=A_{L}(g \circ \gamma)$ for all piecewise $\mathcal{C}^{1}$ curve $\gamma:[a, b] \rightarrow M$. In particular extremal curves of $L$ are sent under $\Gamma_{H}$ to extremal curves, showing that $\mathrm{d} g: T M \rightarrow T M$ also preserves the Euler-Lagrange flow.

If $\mu$ is an invariant measure, then $\mathrm{d} g^{*} \mu$ is also invariant. Moreover, if $\mu$ is minimizing $\mathrm{d} g^{*} \mu$ is also minimizing since

$$
\int_{T M} L \mathrm{~d}\left(\mathrm{~d} g^{*} \mu\right)=\int_{T M} L \circ \mathrm{d} g \mathrm{~d} \mu=\int_{T M} L \mathrm{~d} \mu .
$$

If $(x, v) \in \operatorname{supp}(\mu)$ for a certain minimizing measure $\mu$, then it is clear that $\left(g(x), \mathrm{d}_{x} g(v)\right) \in \operatorname{supp}\left(\mathrm{d}\left(\mathrm{g}^{-1}\right)^{*} \mu\right)$. This proves that $\mathrm{d} g(\widetilde{\mathcal{M}})=\widetilde{\mathcal{M}}$, because $\mathrm{d} g$ is an homeomorphism. Finally, one has that for all $g \in \Gamma_{H}$,

$$
g(\mathcal{M})=g(\pi(\widetilde{\mathcal{M}}))=\pi(\mathrm{d} g(\widetilde{\mathcal{M}}))=\pi(\widetilde{\mathcal{M}})=\mathcal{M}
$$

Let us now consider two solutions $u$ and $u_{0}$ in $\mathcal{S}_{-}$and note $v=u-u_{0}$ their difference; by the last lines of the previous section, both solutions are differentiable at every point $x$ in $\mathcal{M}$ and their derivative must be equal to the Legendre transform of the unique vector in $\widetilde{\mathcal{M}}$ that projects on $x$. Then $v$ is differentiable in all points of $\mathcal{M}$ and at these points $\mathrm{d}_{x} v=\mathrm{d}_{x} u-\mathrm{d}_{x} u_{0}=0$. By Proposition 5 , the orbits by $\Gamma_{H}^{0}$ of each point in $\mathcal{M}$ are closed submanifolds of $M$; as $\mathcal{M}$ contains the orbits of its points, we have, if $x \in \mathcal{M}$ and $V_{x}=\Gamma_{H}^{0}(x)$ is the orbit of $x$, that $V_{x} \subset \mathcal{M}$, and then $d\left(v_{\mid V_{x}}\right)=0$. As $V_{x}$ is a connected submanifold, $v$ must be constant on $V_{x}$. That is to say, two arbitrary solutions differ by a constant on the orbits contained in $\mathcal{M}$. But we know (Lemma 9, see also [5] for a more general statement) that there exists solutions $\mathcal{S}_{-}\left(\right.$or $\left.\mathcal{S}_{+}\right)$ invariant by $\Gamma_{H}$. We therefore conclude that every weak KAM solution is invariant by $\Gamma_{H}^{0}$ on $\mathcal{M}$.

We still have to show the invariance outside $\mathcal{M}$. Let us fix a solution $u$ in $\mathcal{S}_{-}$, an element $g$ of $\Gamma_{H}^{0}$ and any point $x$ of $M$. We will show that $u(g(x))=u(x)$. Given $\epsilon>0$, we choose $\delta>0$ so that for all $x, x^{\prime} \in M$ with $d\left(x, x^{\prime}\right)<\delta$ we have at the same time

$$
\left|u(x)-u\left(x^{\prime}\right)\right|<\epsilon \text { and }\left|u(g(x))-u\left(g\left(x^{\prime}\right)\right)\right|<\epsilon .
$$

Let us now take the curve $\gamma_{x}$ given by Proposition 4 , and $y$ in its $\alpha$-limit set, which is contained in $\mathcal{M}$, whose existence we have already seen; be as well $t<0$ so that $d\left(\gamma_{x}(t), y\right)<\delta$. To simplify the notation we will write $\gamma=\gamma_{x \mid[t, 0]}$ and $z=\gamma(t)$. We then have, through definition from $\gamma$ and because of the domination of $u$ by $L+c(L)$, the following relations:

$$
u(x)-u(z)=A_{L}(\gamma)-c(L) t, \quad \text { and }
$$

BULlETiN DE LA SOCIÉtÉ MATHÉMATIQUE DE FRANCE 


$$
u(g(x))-u(g(z)) \leq A_{L}(g \circ \gamma)-c(L) t=A_{L}(\gamma)-c(L) t
$$

then

$$
u(g(x))-u(x) \leq u(g(z))-u(z) .
$$

Moreover, we have $u(g(y))=u(y)$, when $y \in \mathcal{M}$. Since $d(y, z)<\delta$, we have

$$
|u(y)-u(z)|<\epsilon \text { and }|u(g(y))-u(g(z))|<\epsilon .
$$

This implies $|u(g(z))-u(z)|<2 \epsilon$, hence $u(g(x))-u(x) \leq 2 \epsilon$. Considering that $\epsilon>0$ is arbitrary, we conclude that

$$
u(g(x)) \leq u(x)
$$

for all $x \in M$ and $g \in \Gamma_{H}^{0}$. If we applies this result to $g^{-1}$, it is clear that we obtain the opposite inequality, which proves the theorem.

\section{The group of transformations $\Gamma_{H}$}

Let us first observe that when $H$ is the Hamiltonian associated to the metric on $M$, i.e. $H(x, p)=\frac{1}{2}\|p\|^{2}$, the group of symmetries of $H$ is nothing but the group of the isometries of $M$. A classic theorem by Myers and Steenrod guarantees then that the group is in fact a Lie group [6]. The aim of this section is to generalize this theorem to the group of symmetries $\Gamma_{H}$ that we have defined. We recall that $\Gamma_{H}$ consists of diffeomorphisms of class $\mathcal{C}^{1}$, and that it is endowed with the topology of uniform convergence.

Proposition 5. - The group of transformations $\Gamma_{H}$ is a compact Lie group.

This is an immediate consequence of the following lemma and of the theorem due to Montgomery [9, Thm. 2, p. 208] which establishes that all compact subgroup of the group of $\mathcal{C}^{1}$ diffeomorphisms of a manifold is a Lie group. The following lemma assure the equicontinuity of the symmetries:

Lemma 6. - There exists $K>0$ such that for all $g \in \Gamma_{H}$ and for all $x \in M$, we have $\left\|\mathrm{d}_{x} g\right\| \leq K$

Proof. - From the superlinearity of $H$ we know that there exists a constant $C_{1}^{*} \in \mathbb{R}$ so that for all $(x, p) \in T^{*} M$,

$$
H(x, p) \geq\|p\|+C_{1}^{*} .
$$

If $g$ is such that $H(g(x), p)=H\left(x, p \circ \mathrm{d}_{x} g\right)$, then

$$
H(g(x), p) \geq\left\|p \circ \mathrm{d}_{x} g\right\|+C_{1}^{*} .
$$

On the other hand, the unitary cotangent bundle of $M$ is compact as $M$ is compact. We deduct that for all $p \in T_{g(x)}^{*} M$ with $\|p\|=1$,

$$
\left\|p \circ \mathrm{d}_{x} g\right\| \leq A_{1}^{*}-C_{1}^{*},
$$

TOME $130-2002-\mathrm{N}^{\mathrm{O}} 4$ 
in which

$$
A_{1}^{*}=\sup \left\{H(x, p) ;(x, p) \in T^{*} M,\|p\|=1\right\} .
$$

This proves that $\left\|\mathrm{d}_{x} g\right\| \leq K=A_{1}^{*}-C_{1}^{*}$, a constant which does not depend on $g$ nor on $x$, as we wanted to prove.

To show that the group is compact, we therefore only need to show that it is closed in $\mathcal{C}^{0}(M, M)$ and use the Ascoli's theorem.

Lemma 7. - The group $\Gamma_{H}$ is closed within $\mathcal{C}^{0}(M, M)$.

Proof. - Let $\delta>0$ be so that for all point $x \in M$, the application $\pi \circ \phi_{\delta}^{L}$ defines a diffeomorphism of the ball $B_{x}(0,1)=\left\{v \in T_{x} M ;\|v\|<1\right\}$ on its image; we will denote by $\varphi_{x}$ this diffeomorphism and $U_{x}$ its image. This choice of $\delta$ is possible since the Euler-Lagrange flow comes from a second order equation on $M$ (this means more precisely that $\mathrm{d} \pi \circ X_{L}=\mathrm{id}_{T M}$ ), and $M$ is compact.

Let $g$ be an element of $\Gamma_{H}$ and $x$ a point in $M$. We know that $\mathrm{d} g$ commutes with the flow $\phi_{t}^{L}$ for all $t \in \mathbb{R}$, as $g$ preserves the extremals of $L$. That is to say, for all $(x, v) \in T M$, and for all $t \in \mathbb{R}$ we have $\phi_{t}^{L}\left(\mathrm{~d}_{x} g(v)\right)=\mathrm{d}_{\pi \phi_{t}^{L}(v)} g\left(\phi_{t}^{L}(v)\right)$ so

$$
\pi \circ \phi_{t}^{L}\left(\mathrm{~d}_{x} g(v)\right)=g\left(\pi \circ \phi_{t}^{L}(v)\right) .
$$

It follows that on a small enough neighbourhood $U \subset U_{x}$ of the point $x$ (which by the previous lemma we can clearly choose independent of $g$ ),

$$
g=\varphi_{g(x)} \circ \mathrm{d}_{x} g \circ \varphi_{x}^{-1} .
$$

Let $\left\{g_{n}\right\} \subset \Gamma_{H}$ be a sequence converging uniformely to $g: M \rightarrow M$. If we fix $x \in M$, extracting a subsequence if necessary,, we can suppose that $\mathrm{d}_{x} g_{n}$ tends to the linear application $\alpha \in L\left(T_{x} M, T_{g(x)} M\right)$. On the neighbourhood $U$ of $x$ the sequence

$$
g_{n}=\varphi_{g_{n}(x)} \circ \mathrm{d}_{x} g_{n} \circ \varphi_{x}^{-1}
$$

converges uniformely towards both $g$ and

$$
\varphi_{g(x)} \circ \alpha \circ \varphi_{x}^{-1} .
$$

This shows that $g$ is $\mathcal{C}^{1}$, and that $g_{n} \rightarrow g$ in the $\mathcal{C}^{1}$ topology. Since $H$ is continuous it follows that $g \in \Gamma_{H}$, which proves the lemma.

Finally, let us remark that we have also proved that the elements of $\Gamma_{H}$ are in fact of class $\mathcal{C}^{\infty}$. It is not hard to see that the proofs which have been given for theorem 1 and his corollary are also valid for Hamiltonians of class $\mathcal{C}^{k}$ with $k \geq 3$. In this case, both the Euler-Lagrange flow and the elements of the group $\Gamma_{H}$ are of class $\mathcal{C}^{k-1}$. In the proof of the last lemma, the fact that the Hamiltonian is at least of class $\mathcal{C}^{3}$ is used to guarantee that the maps $\pi \circ \phi_{\delta}^{L}$ are indeed diffeomorphisms. 


\section{Invariant means}

We will now prove the existence of invariant solutions, a fact that we used in the proof of Theorem 1. As we have already said, even if in [5] the existence for Hamiltonians on not necessarily compact manifolds is established, we include here a proof in the compact case. Essentially, we shall prove that the vector space formed by $\Gamma_{H}$-invariant functions is stable under the Lax-Oleinik semigroups, and that they must have a fixed point in the intersection of this space with the convex set of functions dominated by $L+c(L)$ which is also stable under the Lax-Oleinik semigroups. To see that this intersection is not empty, we have to take invariant means of dominated functions. This same averaging technique, proves as is well known that each cohomology class contains invariant closed forms; once this will be done, we will be able to give the proof of Corollary 2.

We denote by $\mathbb{M}$ the invariant mean on the space of continuous functions defined on $\Gamma_{H}$ induced by the normalized Haar integral:

$$
\mathbb{M}: \mathcal{C}^{0}\left(\Gamma_{H}, \mathbb{R}\right) \longrightarrow \mathbb{R}, \quad \mathbb{M}(\psi)=\int_{\Gamma_{H}} \psi(g) \mathrm{d} \lambda(g),
$$

where $\lambda$ is the left invariant Haar measure on $\Gamma_{H}$.

If we take a continuous function on $M$ and we average with $\mathbb{M}$ their restrictions to each orbit of the $\Gamma_{H}$-action, it is clear that we obtain an invariant continuous function on $M$; we shall also denote by $\mathbb{M}$ the operator on $\mathcal{C}^{0}(M, \mathbb{R})$ thus defined. That is, for a fixed function $u \in \mathcal{C}^{0}(M, \mathbb{R})$, and $x \in M$,

$$
\mathbb{M} u(x)=\int_{\Gamma_{H}} u(g(x)) \mathrm{d} \lambda(g) .
$$

Lemma 8. - For each $c \in \mathbb{R}$, the convex set of functions dominated by $L+c$ is stable under the invariant mean operator $\mathbb{M}$.

Proof. — Fix $c \in \mathbb{R}$, and take any function $u \prec L+c$. Let $\gamma:[a, b] \rightarrow M$ be a piecewise $\mathcal{C}^{1}$ curve. If we apply the domination of $u$ to a translated of $\gamma$ by a symmetry $g$ in $\Gamma_{H}$, we have

$$
u(g(\gamma(b)))-u(g(\gamma(a))) \leq A_{L}(g \circ \gamma)+c(b-a) .
$$

But $A_{L}(g \circ \gamma)=A_{L}(\gamma)$ for all $g$ as the action is preserved by symmetries. Meanning these inequalities we obtain

$$
\begin{aligned}
\mathbb{M} u(\gamma(b))-\mathbb{M} u(\gamma(a)) & =\int_{\Gamma_{H}} u(g(\gamma(b)))-u(g(\gamma(a))) \mathrm{d} \lambda \\
& \leq A_{L}(\gamma)+c(b-a),
\end{aligned}
$$

which proves well that $\mathbb{M} u \prec L+c$.

LEMMA 9. - The sets of weak KAM solutions $\mathcal{S}_{-}$and $\mathcal{S}_{+}$both contain $\Gamma_{H^{-}}$ invariant functions.

TOME $130-2002-\mathrm{N}^{\mathrm{O}} 4$ 
Proof. - Let us remember that $\mathbb{H}$ is the convex subset of $\mathcal{C}^{0}(M, \mathbb{R})$ formed by the $L+c(L)$ dominated functions; let us name $\mathcal{I}$ the linear space of $\Gamma_{H}$-invariant functions, and we define

$$
\mathbb{H}_{\text {inv }}=\mathbb{H} \cap \mathcal{I} \text {. }
$$

By the previous lemma, this intersection is not empty, since $\mathbb{H}$ is itself not empty. If we take the quotient of $\mathbb{H}_{\text {inv }}$ by the space of constant functions, we obtain a non empty convex set $\widehat{\mathbb{H}}_{\text {inv }} \subset \widehat{\mathbb{H}}$. Since $\mathcal{I}$ is closed in $\mathcal{C}^{0}(M, \mathbb{R})$ and $\widehat{\mathbb{H}}$ is compact and convex, we obtain that $\widehat{\mathbb{H}}_{\text {inv }}$ is also a compact and non-empty convex subset of $\widehat{\mathcal{C}^{0}}(M, \mathbb{R})$, the quotient of the space of continuous functions by the subspace of constant functions.

Moreover, the Lax-Oleinik semigroups preserve the space of invariant functions: if $t \geq 0$ and $u \in \mathcal{I}$, then once $x \in M$ and $g \in \Gamma_{H}$ are fixed, we know that for all piecewise $\mathcal{C}^{1}$ curve $\gamma:[0, t] \rightarrow M$ with $\gamma(t)=x$,

$$
u(g \circ \gamma(0))+A_{L}(g \circ \gamma)=u(\gamma(0))+A_{L}(\gamma) .
$$

Taking the infimum over all these curves, it results that $T_{t}^{-} u(g(x)) \leq T_{t}^{-} u(x)$, and consequently

$$
T_{t}^{-} u(x)=T_{t}^{-} u\left(g^{-1} g(x)\right) \leq T_{t}^{-} u(g(x)) .
$$

We have then proved that $T_{t}^{-} u \in \mathcal{I}$.

Therefore, the quotient semigroup $\widehat{T}_{t}^{-}$leaves $\widehat{\mathbb{H}}_{\text {inv }}$ invariant. Its continuity is deduced from the one of $T_{t}^{-}$. Applying the theorem of Schauder and Tykhonov we obtain a common fixed point in $\widehat{\mathbb{H}}_{i n v}$ to the whole semi-group, that is to say, a class $\hat{u} \in \widehat{\mathbb{H}}_{\text {inv }}$ such that $\widehat{T}_{t}^{-} \hat{u}=\hat{u}$ for all $t \in \mathbb{R}$. It is clear that any function in this class is an invariant weak KAM solution.

LEMmA 10. - Let $\Omega \in H^{1}(M, \mathbb{R})$ be a cohomology class. There exists a 1 form $\omega_{0}$ in $\Omega$ which is invariant by the action of $\Gamma_{H}^{0}$.

Proof. - The connectedness of $\Gamma_{H}^{0}$ guarantees that all its elements are isotopic to the identity map on $M$; consequently, for all $g \in \Gamma_{H}^{0}$ and all closed form $\omega$, we have that the pull-back of $\omega$ by $g$, which was noted $g^{*} \omega$, is homotopic to $\omega$. In particular, they must be cohomologous. To say this in a more suitable way, the affine action of $\Gamma_{H}^{0}$ on the space of closed forms preserves the cohomology classes. As $\Gamma_{H}^{0}$ is compact, and cohomology classes are also affine spaces, each one of these cohomology classes must have at least one point fixed under $\Gamma_{H}^{0}$, i.e. an invariant form. For 1-form $\omega \in \Omega$ we can give the fixed point explicitly, using the Haar measure, this time normalized on $\Gamma_{H}^{0}$ :

$$
\omega_{0}=\frac{1}{\lambda\left(\Gamma_{H}^{0}\right)} \int_{\Gamma_{H}^{0}} g^{*} \omega \mathrm{d} \lambda(g) .
$$

The form $\omega_{0}$ is in $\Omega$ and clearly it verifies $g^{*} \omega_{0}=\omega_{0}$ for all $g \in \Gamma_{H}^{0}$. 
Proof of Corollary 2. - The graph of a $\mathcal{C}^{1}$ section $\omega: M \rightarrow T^{*} M$ is a Lagrangian submanifold for the standard symplectic form of $T^{*} M$ if and only if $\omega$ is a closed 1-form on $M$. Moreover, this submanifold is invariant by the flow $\phi_{t}^{H}$, if and only if $H \circ \omega$ is constant. If $\Omega=[\omega] \in H^{1}(M, \mathbb{R})$ is the cohomology class of $\omega$, and $\omega_{0}$ the 1 -form in the same class given by the previous lemma, one has that $\omega-\omega_{0}=\mathrm{d} u$ for a certain differentiable function $u: M \rightarrow \mathbb{R}$. The invariance of $\omega$ by the Hamiltonian flow of $H$ can then be written

$$
\forall x \in M, \quad H_{0}\left(x, \mathrm{~d}_{x} u\right)=c
$$

where we have defined $H_{0}(x, p)=H\left(x, p+\omega_{0}(x)\right)$. It is easy to see that $H_{0}$ is also convex and superlinear; moreover, $H_{0}$ define the same Hamiltonian flow as $H$, since $\mathrm{d} \omega_{0}=0$. So we can apply theorem 1 to obtain that $\mathrm{d} u$ is $\Gamma_{H_{0}}^{0}$ invariant; but $\Gamma_{H_{0}}^{0}=\Gamma_{H}^{0}$ as $\omega_{0}$ is $\Gamma_{H}^{0}$-invariant.

\section{The spherical pendulum}

We give here an example where the group of symetries of the system is large. In that case, using the results obtained, we find all the weak KAM solutions by integration. Let us begin by considering the $n$-dimensional sphere $\mathbb{S}^{n}$ naturally embedded in $\mathbb{R}^{n+1}$ and the potential $U: \mathbb{S}^{n} \rightarrow \mathbb{R}$ which associates to each point its last coordinate; that is to say,

$$
U\left(x_{1}, \ldots, x_{n+1}\right)=x_{n+1} .
$$

We will study the weak KAM solutions of the mechanical Hamiltonian which describes the motion of a punctual mass forced to move around on the sphere under the action of the potential $U$. More precisely,

$$
H(x, p)=\frac{1}{2}\|p\|^{2}+U(x) .
$$

We observe that the symmetries of this Hamiltonian are the restrictions to $\mathbb{S}^{n}$ of the orthogonal transformations of $\mathbb{R}^{n+1}$ which fix the last coordinate. The group $\Gamma_{H}$ is then naturally identified to the orthogonal group $\mathrm{O}(n)$, and $\Gamma_{H}^{0}$ to the group $\mathrm{SO}(n)$. The associated Lagrangian is

$$
L(x, v)=\frac{1}{2}\|v\|^{2}-U(x) .
$$

So, its critical value is $c(L)=1$, and the unique minimizing measure is supported on the point $(N, 0) \in T \mathbb{S}^{n}$, where $N$ designates the point of the sphere where $U$ achieves its maximum. The orbits by the action of $\Gamma_{H}^{0}$, coincides with the potential level sets, i.e. the $(n-1)$-dimensional spheres obtained as intersection of $\mathbb{S}^{n}$ with the horizontal hyperplanes $x_{n+1}=k$. As we know, weak KAM solution are determined by its values on the Mather set, so in this case, as $\mathcal{M}=\{N\}$, we have a unique solution in $\mathcal{S}_{-}$modulo an additive constant.

TOME $130-2002-\mathrm{N}^{\mathrm{O}} 4$ 
In order to determine it, we shall call $u$ this solution and we shall assume that $u(N)=0$.

Let $\gamma_{x}:(-\infty, 0]$ be an extremal curve of the Lagrangian, associated to a certain point of $x \in \mathbb{S}^{n}$ by proposition 4 . The fact that $u$ is differentiable in $\gamma_{x}(t)$ with $t<0$ follows the same proposition. In particular, we must have that $\dot{\gamma}_{x}(t)$ is orthogonal to the kernel of $\mathrm{d}_{x} u$ for all $t<0$. Now we can apply Theorem 1 , and deduce that the kernel of $\mathrm{d}_{x} u$ is tangent to the level sets of $U$. Therefore, the curve $\gamma_{x}$ must be contained in the vertical plane generated by $x$ and $N$. That means that $\gamma_{x}$ corresponds to a trajectory of the pendulum on the circle determined by this plane and the sphere. We also have that $\gamma_{x}(t) \rightarrow N$ when $t \rightarrow-\infty$, which proves the differentiability of $u$ in the set $\mathbb{S}^{n}-\{-N\}$. On this set, the Hamilton-Jacobi equation can be written

$$
\left\|\mathrm{d}_{x} u\right\|=\sqrt{2-2 U(x)}
$$

which permits, reparametrizing $\gamma_{x}$ by its last coordinate, to calculate $u$ explicitly by integration.

We conclude that the only weak KAM solutions are given by

$$
u_{ \pm}\left(x_{1}, \ldots, x_{n+1}\right)=u(N) \pm \int_{x_{n+1}}^{1} \sqrt{\frac{2-2 s}{1-s^{2}}} \mathrm{~d} s .
$$

Moreover, there are no differentiable solutions of the Hamilton-Jacobi equation, and no Lagrangian section is preserved by the Hamiltonian flow of this system.

\section{Other examples}

Finally, it is convenient to observe that these results cannot be extended to non compact manifolds, or to Lagrangian submanifolds which are not necessarily graphs. To see this, it is sufficient to consider the following examples; in both cases, the considered Hamiltonian is the corresponding one to the Riemannian metric, its flow the geodesic flow, and so, its critical value is $c(L)=0$.

In a non compact manifold, we can have global solutions of the HamiltonJacobi equation for values of the constant greater than the critical value. In $\mathbb{R}^{n}$, the equation is written $\left\|\mathrm{d}_{x} u\right\|=c$, and each affine function $u: \mathbb{R}^{n} \rightarrow \mathbb{R}$ is a global solution. Except the constant functions (i.e. the solutions for the value of $c=0$ ), they are not invariant under isometries. This is essentially the same example that we could give on $\mathbb{H}^{n}$, the hyperbolic space, Busemann's functions playing the role of affine functions.

On the other hand, Corollary 2 is false for general Lagrangian submanifolds of $T^{*} M$. Let us consider two opposed points $x$ and $-x$ in $\mathbb{S}^{n} \subset \mathbb{R}^{n+1}$, and let us define the functions

$$
u_{+}=d(., x) \quad \text { and } \quad u_{-}=d(.,-x),
$$

BULLETIN DE LA SOCIÉtÉ MATHÉMATIQUE DE FRANCE 
where $d$ is the Riemannian distance on $\mathbb{S}^{n}$. If we call $U \subset \mathbb{S}^{n}$ the complementary open set of $\{x,-x\}$, we have that these two solutions are differentiable on $U$, therefore their derivatives define two Lagrangian graphs in $T^{*} U$, that we shall denote $G_{+}$and $G_{-}$, both diffeomorphic to the product $\mathbb{S}^{n-1} \times(0,1)$. Let also

$$
S_{+}=\left\{p \in T_{x}^{*} \mathbb{S}^{n} ;\|p\|=1\right\} \quad \text { and } \quad S_{-}=\left\{p \in T_{-x}^{*} \mathbb{S}^{n} ;\|p\|=1\right\} .
$$

We define now $N \subset T^{*} \mathbb{S}^{n}$ as the union of these two graphs, and the two spheres $S_{+}$and $S_{-}$. If we observe that $\mathcal{L}^{-1}(N) \subset T \mathbb{S}^{n}$ is the set of all unitary tangent vectors which define geodesics passing by $x$ and $-x$, we see that $N$ is an embedded submanifold of $T^{*} \mathbb{S}^{n}$, diffeomorphic to the product $\mathbb{S}^{n-1} \times \mathbb{S}^{1}$, and preserved by the Hamiltonian flow. We observe that for $t=\frac{1}{2} \pi$, the flow $\phi_{t}^{H}$ sends $G_{+}$and $G_{-}$into open neighborhoods in $N$ of the spheres $S_{+}$and $S_{-}$. As the Hamiltonian flow preserves the symplectic structure of the cotangent bundle, we have proved that $N$ is Lagrangian. Clearly, $N$ is not invariant under the identity component of the isometry group of $\mathbb{S}^{n}$.

\section{BIBLIOGRAPHY}

[1] Contreras (G.), Delgado (J.) \& Iturriaga (R.) - Lagrangian flows: the dynamics of globally minimizing orbits, II, Bol. Soc. Bras. Mat., t. 28 (1997), no. 2, pp. 155-196.

[2] FAthi (A.) - Solutions KAM faibles conjugués et barrières de Peierls, C. R. Acad. Sci. Paris, Série I, t. 325 (1997), pp. 649-652.

[3] _ Théorème KAM faible et théorie de Mather sur les systèmes lagrangiens, C. R. Acad. Sci. Paris, Série I, t. 324 (1997), pp. 1043-1046.

[4] — Weak KAM Theorem in Lagrangian Dynamics, Preprint, 2000.

[5] Fathi (A.) \& Maderna (E.) - Weak KAM Theorem on Non Compact Manifolds, Preprint, 2000.

[6] Kobayashi (S.) - Transformation Groups in Differential Geometry, Springer-Verlag, 1995, reprint of the 1972 ed.

[7] MAÑ́ (R.) - Lagrangian flows: the dynamics of globally minimizing orbits, Bol. Soc. Bras. Mat., t. 28 (1997), no. 2, pp. 141-153.

[8] Mather (J.N.) - Action minimizing measures for positive definite Lagrangian systems, Math. Z., t. 207 (1991), pp. 169-207.

[9] Montgomery (D.) \& ZipPin (L.) - Transformation Groups, Interscience Tracts, vol. 1, J. Wiley \& Sons, 1955.

[10] Paternain (G.) \& Paternain (M.) - Critical values of autonomous Lagrangian systems, Comment. Math. Helvetici, t. 72 (1997), pp. 481-499.

[11] Ziemer (W.) - Weakly Differentiable Functions, Springer-Verlag, 1989. 